\title{
AUTOMATIC SYSTEM FOR FOLLOW-UP OF LEAF AREA GROWTH INDIVIDUALIZED BY PLANTS
}

\section{Thiago da Silva Siqueira*, Felipe Ferreira Bocca, Luiz Henrique A. Rodrigues.}

\begin{abstract}
With the advancement of technology and the low cost of equipment, the possibility to analyze and accompany vegetative vigor in real time is the reality. The vigor is related with the leaf area index, which is a metric obtained by a relation between the leaf area and the soil area occupied by the plant. This research had as objective create an automated system of low cost for acquisition and analysis of images, building an accompaniment of the leaf area index of individualized plants.
\end{abstract}

\section{Key words:}

Computer vision, Image analysis, Individual Segmentation.

\section{Introduction}

The leaf area index (LAl) is an important parameter for the study of plants development. This metric is a dimensionless value that relates the leaf area (area of leaves present in the canopy) with the soil area occupied by the plant [1]. The LAI can be used as an indicator of the vegetative vigor and availability of water for the plant, besides being a key parameter for simulations of ecosystem productivity [2].

Monitoring of various plants requires rapid data acquisition and analysis for decision making. With this need and the advance of equipments available in the market, this research aimed to create an automated low-cost system to image acquisition and analysis, by following up the leaf area index of individualized plants.

\section{Results and Discussion}

One image per production day (at noon) was captured, with a camera $\mathrm{Pi}$ NolR, connected to a single board computer (Raspberry Pi).

With the captured images, a routine in Python was built for the segmentation and calculation of the leaf area. Figure 1 presents an image of the pilot production and a representation of the marking of the groups by the routine.
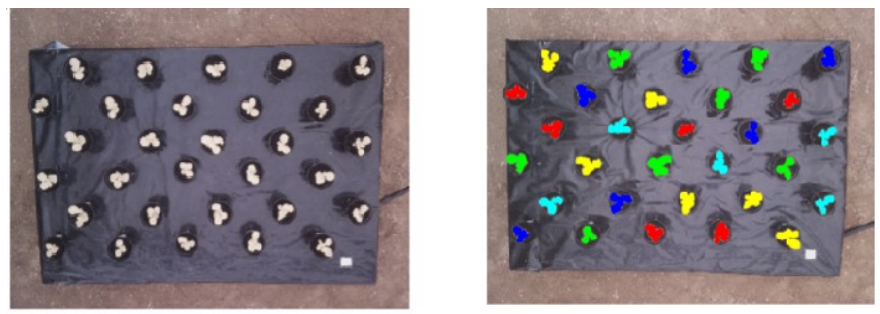

Figure 1. Comparative between image and segmentation.

Excessive heat, responsible for deformation of the dark mantle used at the base and the noonday sun to set during the capture of the images, have compromised the segmentation of some images due to the high incidence of reflection below the canopy.

Hence, only 12 images of the 18-day experiment were used to create the growth curves of the plant's leaf area.
Figure 2 presents the curves with the growth average of each lettuce group over time.

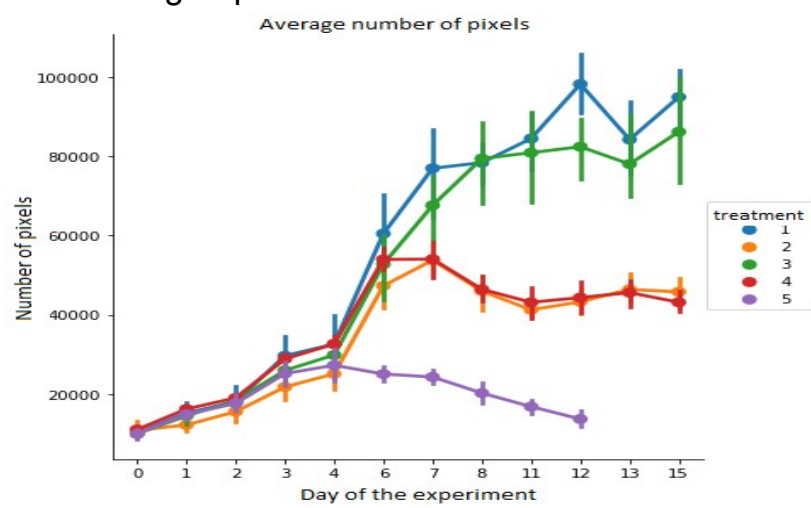

Picture 2. Average growth curve by groups.

At the beginning of the experiment, all treatments had similar results due to the initial state of plants pots saturation. After day 4, group 5 (without the addition of water/nutrients) lost part of the water to the environment, thus reducing its leaf area. Something similar happens on day 7 for the plants of groups 2 and 4 , where they present a limitation of growth compared to groups 1 and 3 , and this is due to the lower availability of water and nutrients for this group.

\section{Conclusions}

By analyzing the preliminary results, it is possible to observe the difference between the growth in the different treatments and points where the plants suffered water stress.

For the next experiments, it is important to change the lower mantle with some matte material. This can help not to lose images due to the reflection. Changing the time of images acquisition should also help.

[1] Jonckheere, Inge, Stefan Fleck, Kris Nackaerts, Bart Muys, Pol Coppin, Marie Weiss, and Frédéric Baret. 2004. "Review of Methods for in Situ Leaf Area Index Determination: Part I. Theories, Sensors and Hemispherical Photography." Agricultural and Forest

Meteorology 121 (1-2): 19-35.

[2] Breda, Nathalie J. J. 2003. "Ground-Based Measurements of Leaf Area Index: A Review of Methods, Instruments and Current

Controversies." Journal of Experimental Botany 54 (392): 2403-17. 